\title{
Ova de Calculo Integral para disminuir la deserción en estudiantes de ingeniería.
}

\section{OVA of Integral Calculation to diminish the desertion in engineering students}

\author{
Fernando Riveros Sanabria, M.Sc ${ }^{1}$, Oscar Agudelo Varela, M.Sc ${ }^{2}$, Elsa Edilma Páez Castro, M.Sc ${ }^{3}$ \\ Universidad de los Llanos ${ }^{123}$, Colombia ${ }^{123}$, friveros@unillanos.edu.co ${ }^{1}$, oscar.agudelo@unillanos.edu.co ${ }^{2}$, \\ elsapcastro@unillanos.edu.co
}

\begin{abstract}
This Virtual Learning Object makes use of information and communication technologies to support the process of teaching Integral Calculus in the engineering programs of the Universidad de los Llanos, in order to reduce student dropout for academic reasons. It is one more option that is presented for teaching-learning in support of the Program of Permanence and Retention Unillanista. Its impact is measurable in the increase of the average performance per semester of Mathematics II in the undergraduate programs of Electronic Engineering and Systems Engineering.
\end{abstract}

Keywords- Virtual Learning Object, Information and Communication Technologies, Teaching-learning process, Integral Calculus, Dropout.

Resumen - Este Objeto Virtual de Aprendizaje hace uso de las tecnologías de la información y las comunicaciones para apoyar el proceso de enseñanza de Cálculo Integral en los programas de Ingeniería de la Universidad de los Llanos, con el objeto de reducir la deserción estudiantil por razones académicas.

Es una opción más que se presenta para la enseñanzaaprendizaje en apoyo al Programa de Permanencia y Retención Unillanista. Su impacto es medible en el aumento del rendimiento promedio por semestre de la asignatura Matemáticas II en los programas de pregrado de Ingeniería Electrónica e Ingeniería de Sistemas.

Palabras Clave - Objeto Virtual de Aprendizaje, Tecnologías de la Información y las Comunicaciones, Proceso de enseñanzaaprendizaje, Cálculo Integral, Deserción.

\section{INTRODUCCIÓN}

La Universidad de los Llanos (Unillanos) a través del Programa de Retención Estudiantil Unillanista (PREU), propende por el fomento de estrategias, proyectos y actividades que contribuyan a generar un mayor ambiente de bienestar en el estudiante de pregrado de la Universidad, principalmente de los estudiantes matriculados en los primeros tres semestres de carrera [1].

El PREU considera, que el desarrollo de las tecnologías de la información y las comunicaciones (TIC) han permitido la producción de recursos académicos basados en aplicaciones interactivas que, dado su carácter dinámico y atractivo, hacen posible la visualización de conceptos abstractos, facilitando su aprendizaje [2].

Investigadores de Unillanos señalan que la mortalidad académica está altamente concentrada en el área de las ciencias básicas y ciencias naturales, principalmente en los primeros tres semestres de las distintas carreras. Para el caso específico de las Ingenierías de Sistemas y Electrónica, se encuentra en los cursos de Matemáticas, Física, Álgebra Lineal, Ecuaciones Diferenciales, Programación y Circuitos [3].

El grupo de Investigación Horizonte Mediático en colaboración con el PREU diseño, desarrollo y desplego un Objeto Virtual de Aprendizaje (OVA) con el propósito de motivar el proceso de aprendizaje de Cálculo Integral en los estudiantes de los programas de Ingeniería de Sistemas y Electrónica establecidos en la Facultad de Ciencias Básicas e Ingeniería de Unillanos [4].

\section{CONSIDERACIONES}

El aprendizaje de Cálculo Integral ha sido en la mayoría de los casos un proceso tedioso y con múltiples dificultades, dada su propia condición de rigurosidad de los conceptos y teorías, como lo establece "Troubles Understanding the Concept of Integral: an Explanation" [5], es así que, lograr llevar estos conceptos a los estudiantes, y que ellos se apropien de los mismos, es un procedimiento con cierta dificultad

La implementación de un OVA requiere estrategias basadas en modelos pedagógicos, aprovechamiento del avance tecnológico disponible, modelos disciplinares y contextuales para facilitar el trabajo del estudiante y el quehacer del docente. Consciente de lo anterior, la Universidad ofrece su infraestructura de TIC para la puesta en funcionamiento de un OVA para el curso Matemáticas II en los temas de Cálculo Integral, para que el estudiante adquiera un nivel de desarrollo de las competencias cognitivas y praxiológicas en esta rama disciplinar. 
El Comité de Estándares de Tecnologías de Aprendizaje define "Un Objeto de Aprendizaje es cualquier entidad, digital o no digital, la cual puede ser usada, re-usada o referenciada durante el aprendizaje apoyada por tecnología [6]. Ejemplos de aprendizajes apoyados por tecnología incluyen sistemas de entrenamiento basados en computador, ambientes de aprendizaje interactivos, sistemas inteligentes de instrucción apoyada por computador, sistemas de aprendizaje a distancia y ambientes de aprendizaje colaborativo".

Las características que debe contener un OVA son las siguientes [7]:

- Reutilización: objeto con capacidad para ser usado en contextos y propósitos educativos diferentes y para adaptarse y combinarse dentro de nuevas secuencias formativas.

- Educatividad: capacidad para generar aprendizaje.

- Interoperabilidad: capacidad para poder integrarse en estructuras y sistemas (plataformas) diferentes.

- Accesibilidad: facilidad para ser identificados, buscados y encontrados, gracias al correspondiente etiquetado a través de diversos descriptores (metadatos) que permitirían la catalogación y almacenamiento en el correspondiente repositorio.

- Durabilidad: vigencia de la información de los objetos, sin necesidad de nuevos diseños.

- Independencia y autonomía: de los objetos con respecto de los sistemas desde los que fueron creados y con sentido propio.

- Generatividad: capacidad para construir contenidos, objetos nuevos derivados de él. Capacidad para ser actualizados o modificados, aumentando sus potencialidades a través de la colaboración.

- Flexibilidad, versatilidad y funcionalidad: capacidad para poder combinarse con diversas propuestas de áreas del saber diferentes.

Una vez revisado el proceso de enseñanza-aprendizaje durante los últimos cuatro (4) periodos académicos de la asignatura Matemáticas II (correspondiente a Cálculo Integral, aplicaciones, sucesiones y series) en los programas de Ingeniería de Sistemas y Electrónica, se estableció que esta asignatura presenta dificulta en la comprensión de ciertos temas para la mayoría de los estudiantes, confirmando [1], los temas en cuestión son:
1. Métodos de integración.

- Anti-derivada.

- Integral indefinida.

- Integración por sustitución.

- Integración por partes.

- Integración por fracciones parciales.

- Integración por sustitución trigonométrica.

2. Integral definida.

- Suma de Riemann (Conceptos previos).

- Integral definida.

- Área bajo la curva.

- Propiedad de la integral definida.

- Teorema del valor medio para integrales.

- Valor promedio.

- Teorema fundamental del cálculo A

- Teorema fundamental del cálculo B

3. Aplicaciones

- Área bajo la curva.

- Área entre funciones

- Sólidos de revolución de discos y arandelas

\section{METODOLOGÍA}

El proceso empleado para el uso del OVA, comprende 3 actores y las interrelaciones entre ellos y esté, los actores son:

- Docente

- Auxiliares o Monitor

- Estudiante

\section{Docente}

Los docentes de los cursos en los cuales los estudiantes tienen dificultades, son invitados por el PREU a una reunión, en la cual se exponen los recursos humanos y tecnológicos con los que cuentan los docentes para apoyar sus clases, estos están en toda libertad de usar o no los recursos en sus actividades académicas.

\section{Auxiliares}

El PREU realiza una convocatoria para seleccionar auxiliares y monitores, para lo cual requiere notas de las asignaturas de cálculo y promedio de carrera. Se seleccionan los estudiantes de ingeniería que tengan las mejores notas. Para ser auxiliar se requiere un promedio más alto. Con los auxiliares y monitores definidos se realiza una reunión para explicarles el uso del OVA y recomendaciones para el manejo con los estudiantes.

\section{Estudiantes}

$16^{\text {th }}$ LACCEI International Multi-Conference for Engineering, Education, and Technology: "Innovation in Education and Inclusion", 18-20 July 2018, Lima, Perú. 
Una semana antes de iniciar el primer semestre, todos los nuevos estudiantes de la Universidad reciben una capacitación orientada por el PREU, en la cual se busca establecer o generar patrones de buen estudio y explicar las herramientas tecnológicas con las que cuenta la Universidad, entre estas el OVA.

La enseñanza tradicional en la cual un docente usa el tablero y el marcador para representar los conceptos del Cálculo Integral, limitan la participación del estudiante, ya que este debe concentrarse en ver el tablero, tomar apuntes y entender lo se está explicando, ver figura 1.

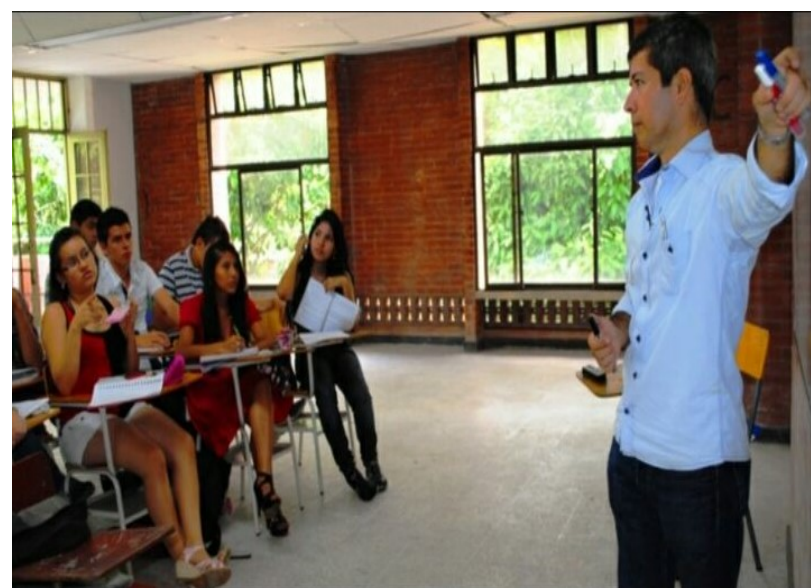

Fig. 1 Docente tradicional, ver el tablero y tomar apuntes

La presunción del triángulo alumno-profesor-contenidos como unidad básica de análisis de los procesos de enseñanza y aprendizaje en contextos virtuales [8][9], es ampliada al incorporar al auxiliar y dar interactividad a los contenidos, lo cual genera cambios en el triángulo.

Durante el primer semestre de liberado el OVA se siguió el siguiente proceso, el docente puede impartir su clase de manera tradicional, sin involucrar el OVA o durante la clase utiliza el OVA como material de apoyo para sus explicaciones o variaciones de ejercicios, ya que cuenta con GeoGebra un software de licencia libre e interactivo, que se compone de Calculadora de Matemáticas con gráficas 3D, Geometría, Cálculo Simbólico, hoja de cálculo y de variados recursos que permiten la generación de simulaciones, lo cual permite al docente el despliegue de graficas fácilmente, ver figura 2. Adicionalmente el docente puede dejar ejercicios para realizar y comprobar en el OVA.

Es de tener en cuenta que algunos estudiantes prefieren por varias razones no preguntar al docente. El auxiliar apoya al docente durante la clase, resolviendo dudas de los talleres, revisando los procesos y respuestas de los ejercicios y algunas otras inquietudes de los estudiantes. Por fuera de la clase, el monitor explica temas, colabora en la resolución de ejercicios y tareas propuestas por el docente, apoyándose y promoviendo el uso del OVA en los estudiantes.

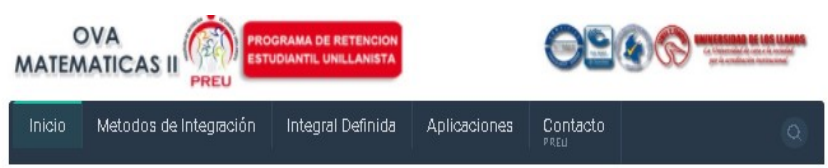

Rotación de discos

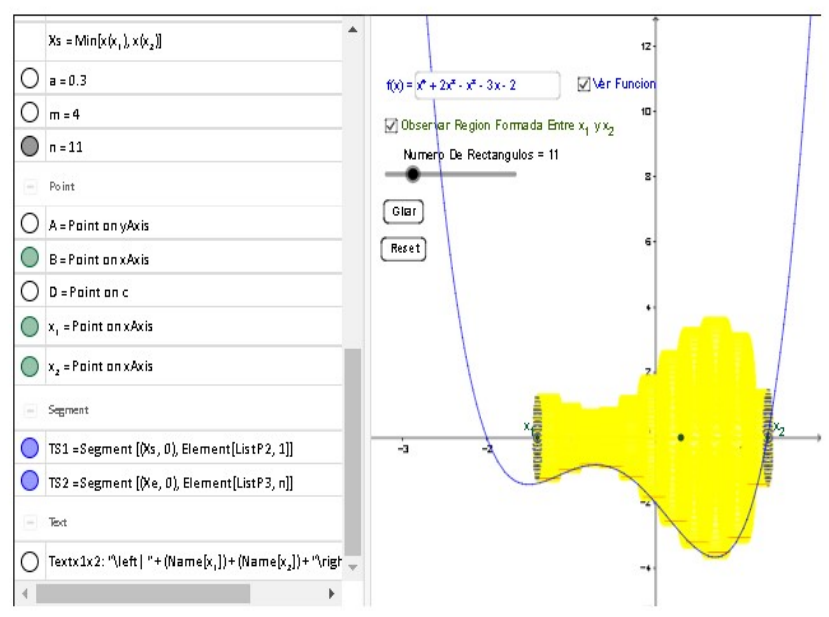

Fig. 2 Simulación con GeoGebra

El OVA fue desarrollado pensado principalmente en los estudiantes, es así, que se busca que el OVA cuente con interfaces agradables, fáciles de usar y recordar, además con acceso web para facilitar su visualización en cualquier dispositivo que se conecte a la Web, ver figura 3.

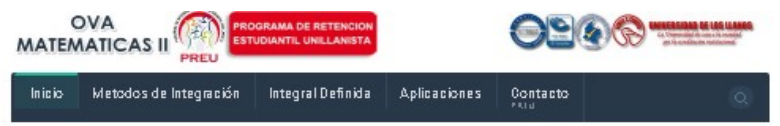

Aplicaciones

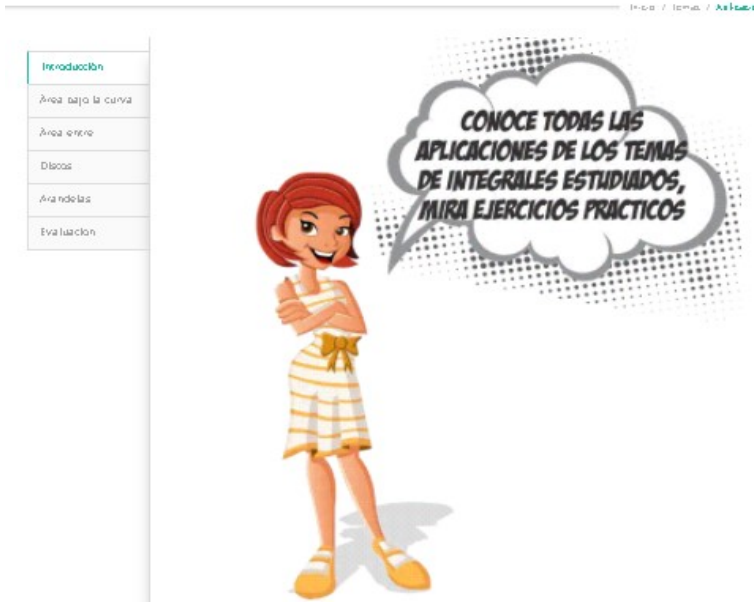

Fig. 3 Interface y colores, tema Aplicaciones 
Los estudiantes disponen del apoyo del docente, el monitor y el OVA, además del recurso tradicional de la biblioteca, para realizar sus repasos o estudios, la Universidad ofrece a los estudiantes y monitores acceso a salas de informática en las cuales pueden usar tableros electrónicos que motivan y facilitan el uso del OVA, ver figuras 4 y 5 .

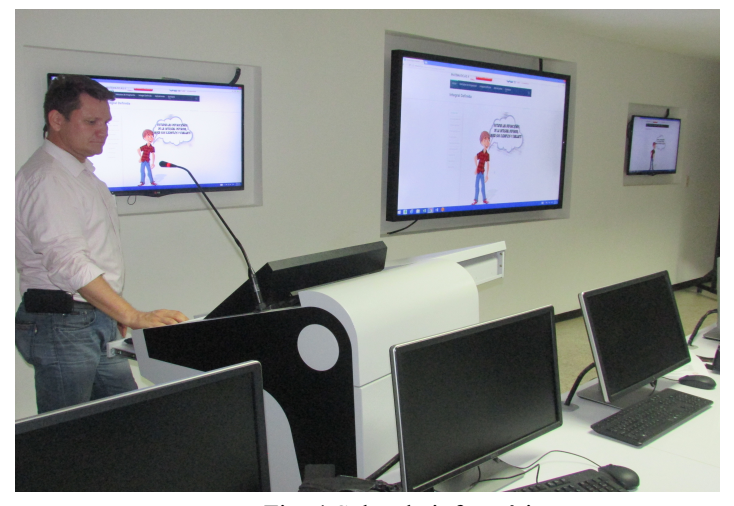

Fig. 4 Salas de informática

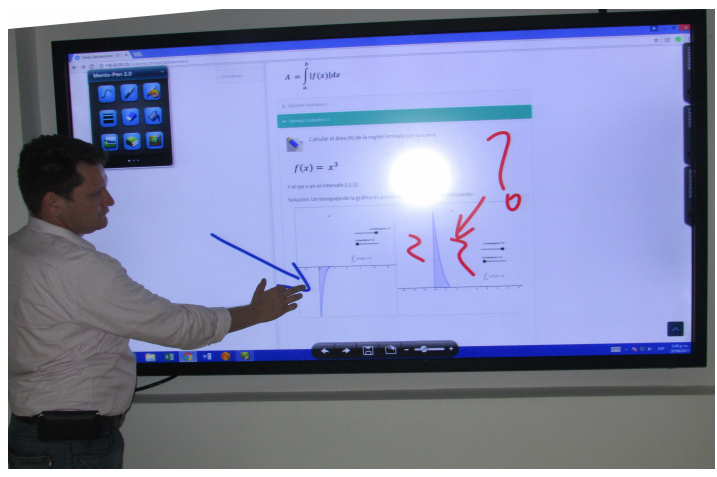

Fig. 5 Uso del tablero digital

El OVA se apoya en el principio de compreder y recordar [11], soportado por las imágenes, animaciones, simulaciones, ejercicio y evaluaciones, que permiten que el estudiante desarrolle su potencial y le otorgan liberta en su aprendizaje. ver figura 6 .

\section{ANÁLISIS DE RESULTADOS}

Al finalizar el semestre se realizaron encuestas de satisfacción en los estudiantes para establecer la percepción en aspectos como: el diseño gráfico, la usabilidad y los contenidos del OVA. A continuación se presentan 2 graficas que ilustran los porcentajes de aceptación del uso de las simulaciones y la suficiencia de la información, ver gráficas. 1 y 2 .

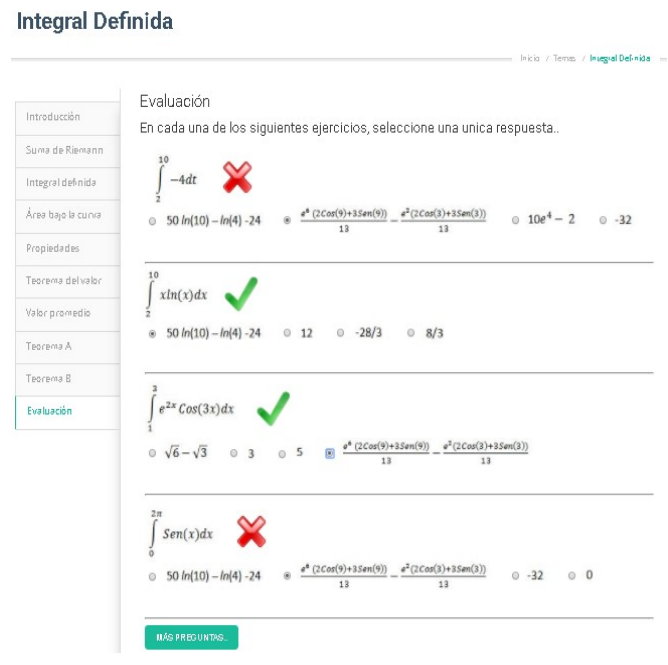

Fig. 6 Interfaz de evaluación

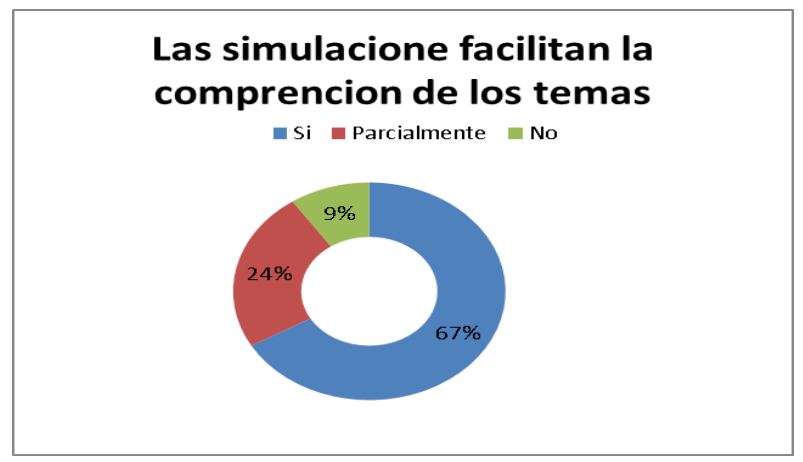

Graf. 1 Pregunta 5 de encuesta de satisfacción

Se observa que el OVA fue bien aceptado por los estudiantes y manejó una correcta relación de las actividades planteadas, sin embargo aún se requiere ampliar y profundizar las temáticas.

\section{¿Considera que éste OVA contiene la información suficiente sobre el tema?}

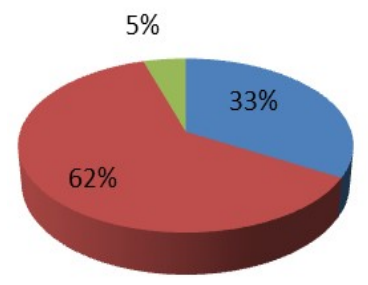

ES COMPLETA
ES PARCIALMENTE COMPLETA

ES INSUFICIENTE

口TRO

Graf. 2 Pregunta 8 de encuesta de satisfacción

Según graficas 3 y 4 aportadas por el PREU [13] se observa que el uso del OVA en los últimos cuatro semestres 
generó un aumento del promedio por semestre de la asignatura Matemáticas II en los programas de Ingeniería de la Facultad (Ingeniería Electrónica e Ingeniería de Sistemas).

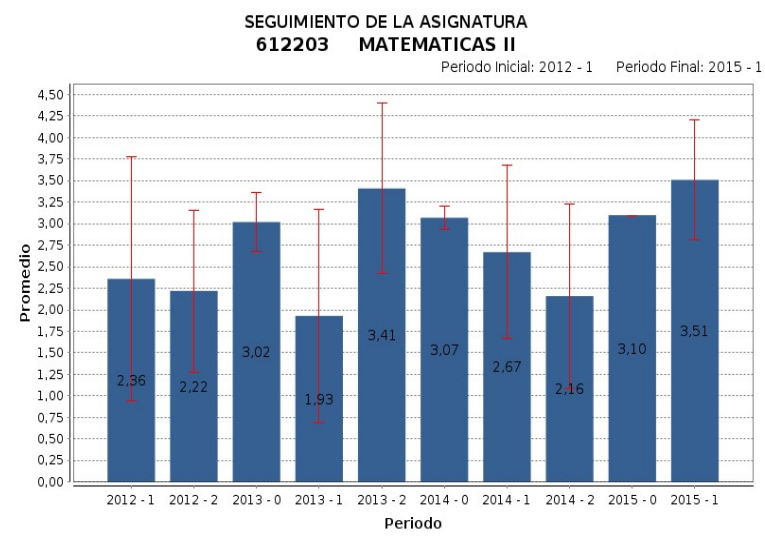

Graf. 3 Histórico del promedio final de la asignatura Matemáticas II del programa de Ingeniería Electrónica

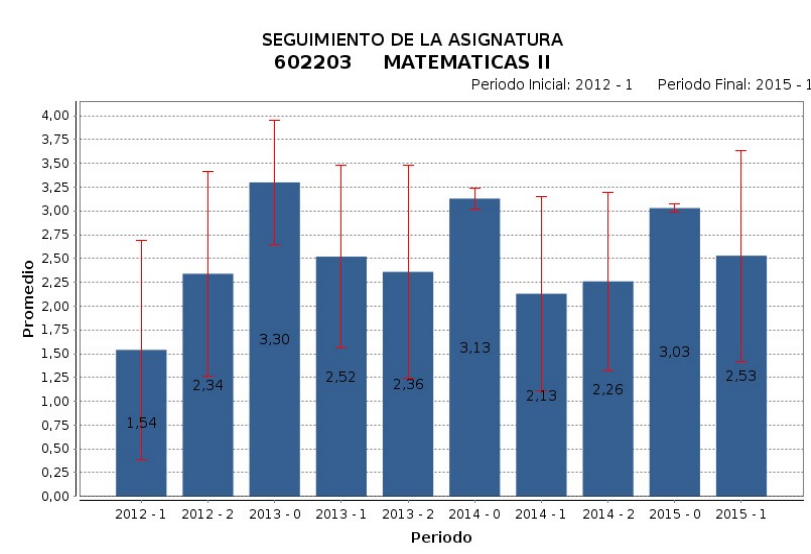

Graf. 4. Histórico del promedio final de la asignatura Matemáticas II del programa de Ingeniería de Sistemas

\section{CONCLUSIONES}

El OVA ofrece al estudiante otra forma de acercarse a la comprensión de un tema de Matemáticas II, con la posibilidad de generar creatividad e interés en ampliar los conceptos vistos.

La Universidad cuenta con una herramienta que permite al estudiante, el desarrollo de habilidad y destreza ligado a cada grado de competencia exigidas.

El PREU reporta un aumento en promedio de las notas de los estudiantes, dado el uso del OVA asociado al apoyo de los monitores.

Los docentes son parte importante del proceso de enseñanza-aprendizaje por lo tanto deben tener capacitación en el manejo del OVA o herramientas tecnológicas para incentivar su uso en los estudiantes.

$16^{\text {th }}$ LACCEI International Multi-Conference for Engineering, Education, and Technology: "Innovation in Education and Inclusion”, 18-20 July 2018, Lima, Perú.
La generación de nuevos espacios que transformen el proceso educativo mediante las TIC, permiten una mejora continua que facilitan la acreditación de los programas.

\section{REFERENCES}

[1] Cartilla Ilustrativa PROGRAMA DE RETENCIÓN ESTUDIANTIL UNILLANISTA PREU, Unillanos, 2013

[2] Alvarez, Teresa. La visualización de conceptos matemáticos y el aprendizaje del electromagnetismo. Latin-American Journal of Physics Education. Vol. 4 Issue 1, p143-148. 2010

[3] Malagón Escobar, Soto Hernández, Pedro Eslava Mocha, La deserción en la Universidad de los Llanos (1998-2004), Orinoquia, 2007.

[4] Agudelo Varela Oscar; Riveros Sanabria Fernando; Páez Castro Elsa, 'Diseño de Materiales Virtuales para el Aprendizaje de Cálculo Integral en Ingeniería con el Propósito de Reducir la Tasa de Deserción Estudiantil en la Universidad de los Llanos. CISCI 2017

[5] J. L. Llorens Fuster y Francisco José Santonja Gómez, "Troubles Understanding the Concept of Integral: an Explanation" Departamento de Matemática Aplicada. Universidad Politécnica de Valencia. Valencia. España, 1997.

[6] Learning Tecnology Standards Committee LTSC http://lsc.ieee.org

[7] García Aretio, L. (2005). Objetos de aprendizaje: características y repositorios. [España]: BENED, 2005. Disponible en: http://www.tecnoeducativos.com/descargas/objetos_virtuales_depare dizaje.pdf

[8] Onrubia, J. (2005, Febrero). Aprender y enseñar en entornos virtuales: actividad conjunta, ayuda pedagógica y construcción del conocimiento. RED. Revista Educación a Distancia, número monográfico II. Consultado el 9 de Febrero 2005 en http://www.um.es/ead/red/M2/

[9] Serrano González-Tejero José Manuel, Pons Parra Rosa María, "La concepción constructivista de la instrucción. Hacia un replanteamiento del triángulo interactivo". Revista mexicana de investigación educativa versión impresa ISSN 1405-6666 RMIE vol.13 no.38 México jul./sep. 2008

[10] https://www.geogebra.org/about

[11] Academia Nacional de Ciencias de Estados Unidos, How People Learn: Brain, Mind, Experience, and School. National Academies Press. 2000.

[12] Oficina del Programa de Retención Unillanista (PREU). http://preu.unillanos.edu.co/. 2016 